\title{
Transport of charged dust grains into the galactic halo
}

\section{Sergey Khoperskov*†}

Institute of Astronomy of the Russian Academy of Sciences, Pyatnitskaya st., 48, 119017

Moscow, Russia

Sternberg Astronomical Institute, Moscow MV Lomonosov State University, Universitetskij pr.,

13, 119992 Moscow, Russia

E-mail: khoperskoveinasan.ru

\section{Yuri Shchekinov}

Southern Federal University, Sorge Str. 5, Rostov-on-Don 344090, Russia

E-mail: vusdstedu.ru

We develop a 3D dynamical model of dust outflows from galactic discs. The outflows are initiated by multiple SN explosions in a magnetized interstellar medium (ISM) with a gravitationally stratified density distribution. Dust grains are treated as particles in cells interacting collisionally with gas, and forced by stellar radiation of the disc and Lorenz force. We show that magnetic field plays a crucial role in accelerating the charged dust grains and expelling them out of the disc: in 10-20 Myr they can be elevated at distances up to $10 \mathrm{kpc}$ above the galactic plane. The dust-to-gas ratio in the outflowing medium varies in the range $5 \cdot 10^{-4}-5 \cdot 10^{-2}$ along the vertical stream. Overall the dust mass loss rate depends on the parameters of ISM and may reach up to $3 \times 10^{-2} \mathrm{M}_{\odot} \mathrm{yr}^{-1}$.

The Life Cycle of Dust in the Universe: Observations, Theory, and Laboratory Experiments - LCDU 2013, 18-22 November 2013

Taipei, Taiwan

\footnotetext{
*Speaker.

${ }^{\dagger}$ RFBR grants 14-02-00604, 14-02-31456

${ }^{\ddagger}$ RFBR grant 12-02-00917
} 


\section{Introduction}

The dominant sources of dust in the Milky Way are the AGB stars [四], supernovae and young stellar objects and cool dense ISM regions [[]]. Stellar winds and jets from YSO supply dust into the nearest interstellar medium where it is supposed to mix with ambient gas. SNe ejecta are also thought to be an efficient source of dust, though it is unclear what fraction of dust survives in the reverse shocks of SNe remnants [[]]. However, it is absolutely clear that the sources of dust concentrate basically within the galactic stellar disc.

At the same time, there are copious evidences of dust extending in the vertical direction up to tens of the scale height of the stellar thin disc [四, [1, 目, 四]. In the radial direction dust may occupy at least twice as large a disc as the stellar one. In addition, more recently evidences of dust present in the intergalactic medium have also appeared in the literature [ [ $]$ ].

In this contribution we briefly describe our results of simulations of dust transport within a 3D $\mathrm{N}$-body/hydrodynamical framework on scales of the galactic halo. Aiming to understand of how the galactic halo and galaxy outskirts are enriched with dust we developed a numerical scenario of dust driven by a combined action of stellar radiation pressure, multiple supernova explosions and Lorenz forces in a magnetized gravitationally stratified ISM.

\section{Model}

We consider a dust-gaseous mixture in a $20 \mathrm{kpc}$ height column based on a $1 \mathrm{kpc} \times 1 \mathrm{kpc}$ square in the plane immersed into the gravitation field of the stellar disc and the dark matter halo. Description of gas dynamics is based on the TVD MUSCL scheme with a $10 \mathrm{pc}$ spatial resolution. Dust grains are treated within a collisionless $N$-body $\left(10^{7}\right.$ probe particles $)$ description. Such an
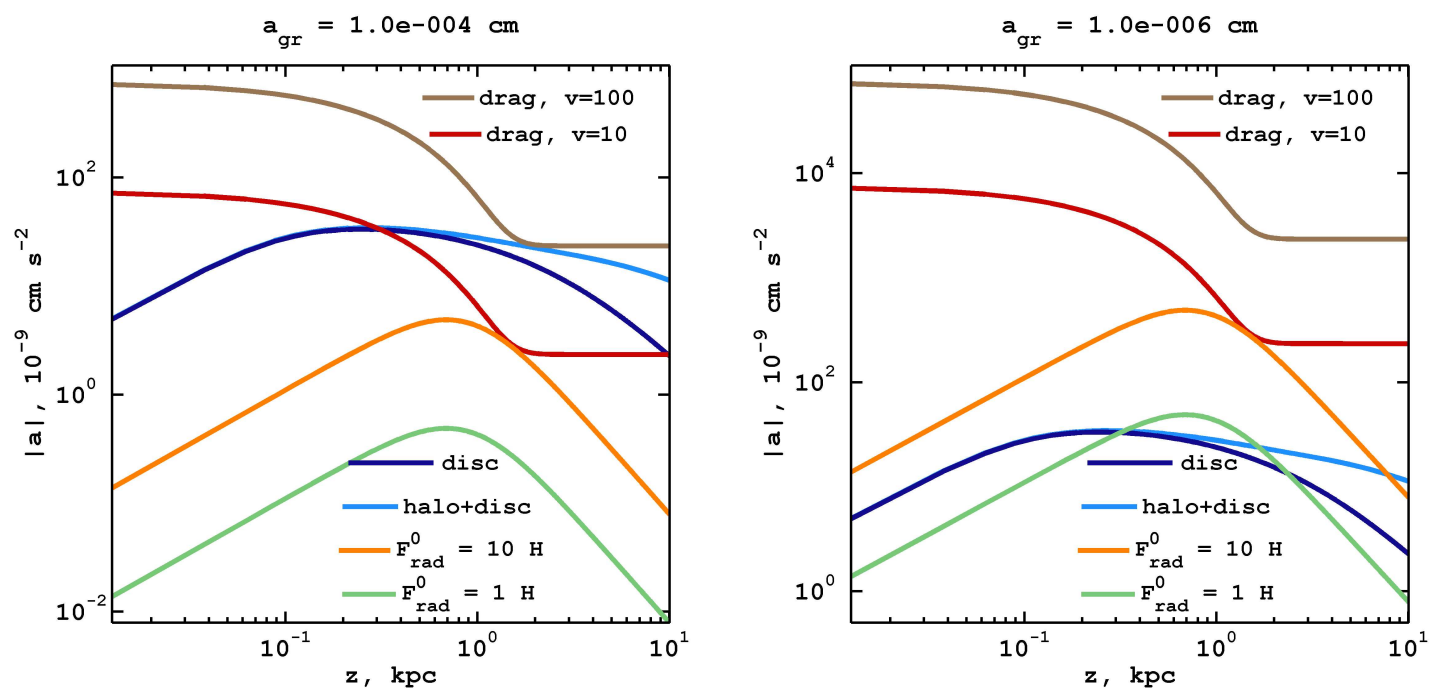

Figure 1: Contributions to the vertical acceleration of dust grains of radius $a=10^{-4} \mathrm{~cm}$ (left panel) and $a=10^{-6} \mathrm{~cm}$ (right panel): gravity from the disc (dark blue), from the disc and the halo (light blue); radiation pressure for 1 (green) and 10 Habing fluxes (orange); drag forces for a grain moving through the gaseous halo with a velocity $v=10 \mathrm{~km} \mathrm{~s}^{-1}$ (red) and $100 \mathrm{~km} \mathrm{~s}^{-1}$ (brown). 

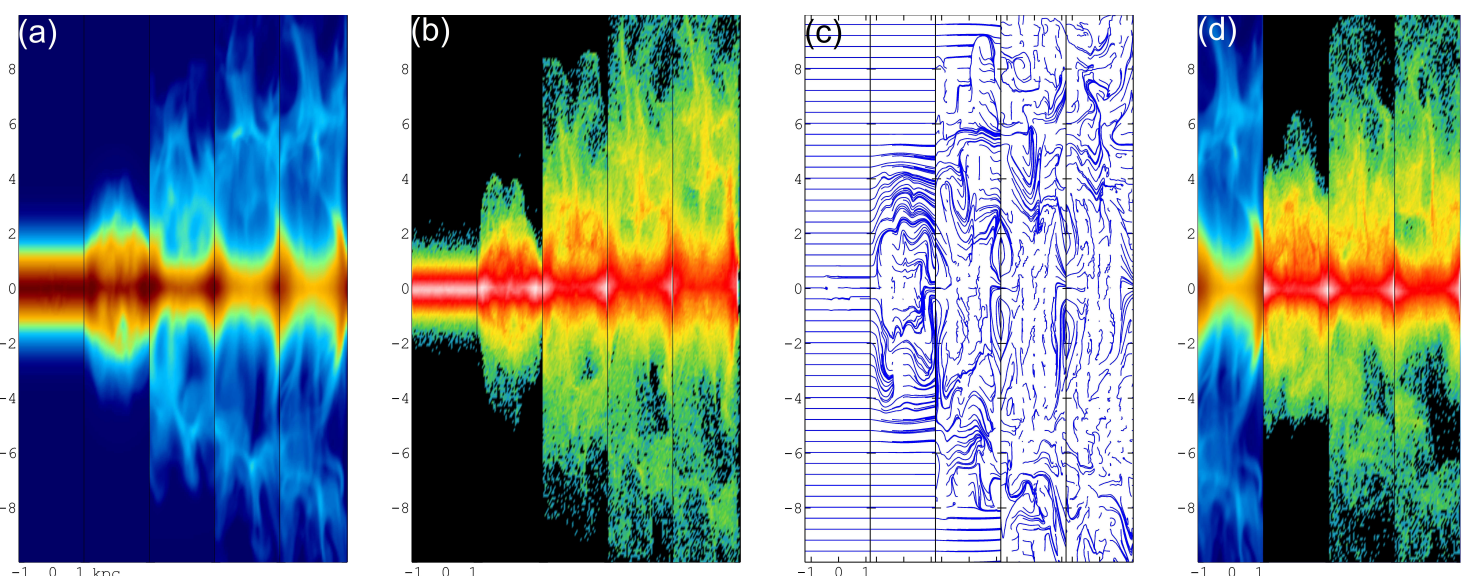

Figure 2: Evolution of gas column density (a), dust column density (b) and magnetic field structure (c) at $t=2 ; 7 ; 11 ; 15$ Myr. Panel (d) is the distribution of gas (left) and dust grains of different sizes $\left(a=10^{-4}\right.$; $10^{-5} ; 10^{-6} \mathrm{~cm}$ - from left to right) at $t=15 \mathrm{Myr}$.

approach allows us to treat separately motions of dust and gas in the presence of magnetic and radiation fields, mutual interaction of dust and gas components is due to the collisional friction: see Figure $\mathbf{~}$.

Initially the gaseous disc is in the hydrostatic equilibrium, with a $\beta=1$ magnetic field parallel to the disc plane; dust grains occupy the $2 \mathrm{kpc}$ layer $(|z|<1 \mathrm{kpc})$ with the standard dust-to-gas ratio 0.01. SN energy $E_{51}=E / 10^{51} \mathrm{erg}$ is injected into the disc with the scale height $h_{\mathrm{sn}} \approx 100 \mathrm{pc}$ and with the current rate for the Milky Way disc of $(370)^{-1} \mathrm{yr}^{-1}$ (type I + type II). Thus, during a $10^{7} \mathrm{yr}$ period we randomly distribute $\approx 2 \cdot 10^{3} \mathrm{SN}$ explosions within the computational domain. Each $\mathrm{SNe}$ is represented by energy $E_{51}=1$ injected into the computational cell instantaneously. Our description of gaseous component is similar to the one developed by de Avillez and Breitschwerdt for modelling a realistic ISM heated by multiple SNe explosions [Q].

\section{Results}

- Supernovae explosions along with radiation pressure from associated stellar population with a flux of $1-10$ Habing drive dust outflows whose overall dynamics depends on gas density and plasma- $\beta$ in the plane. Gas is expelled only by the shock waves from SNe.

- An asymmetry between positive and negative altitudes is clearly seen: Fig. $\square(a, b, c)$. This is due to a particular choice of the random spatial distribution of $\mathrm{SNe}$ explosions.

- Due to a predominance of gravity $\propto a^{3}$ over collisional coupling and radiation force $\propto a^{2}$ larger particles are elevated slower than the smaller ones, as seen from Fig. 1 (d).

- Even though initially dust and gas are assumed to be well mixed, during their elevation they separate such that the dust-to-gas ratio varies from point to point: Fig. [] (left).

- There are two regimes of dust outflows: 1) fast motion with the rate $10^{-3}-3 \cdot 10^{-2} \mathrm{M}_{\odot} \mathrm{yr}^{-1}$, and 2) nearly constant dust mass loss rate $\sim 4 \cdot 10^{-5}-3 \cdot 10^{-3} \mathrm{M}_{\odot} \mathrm{yr}^{-1}$ : Figure 3 (right). 

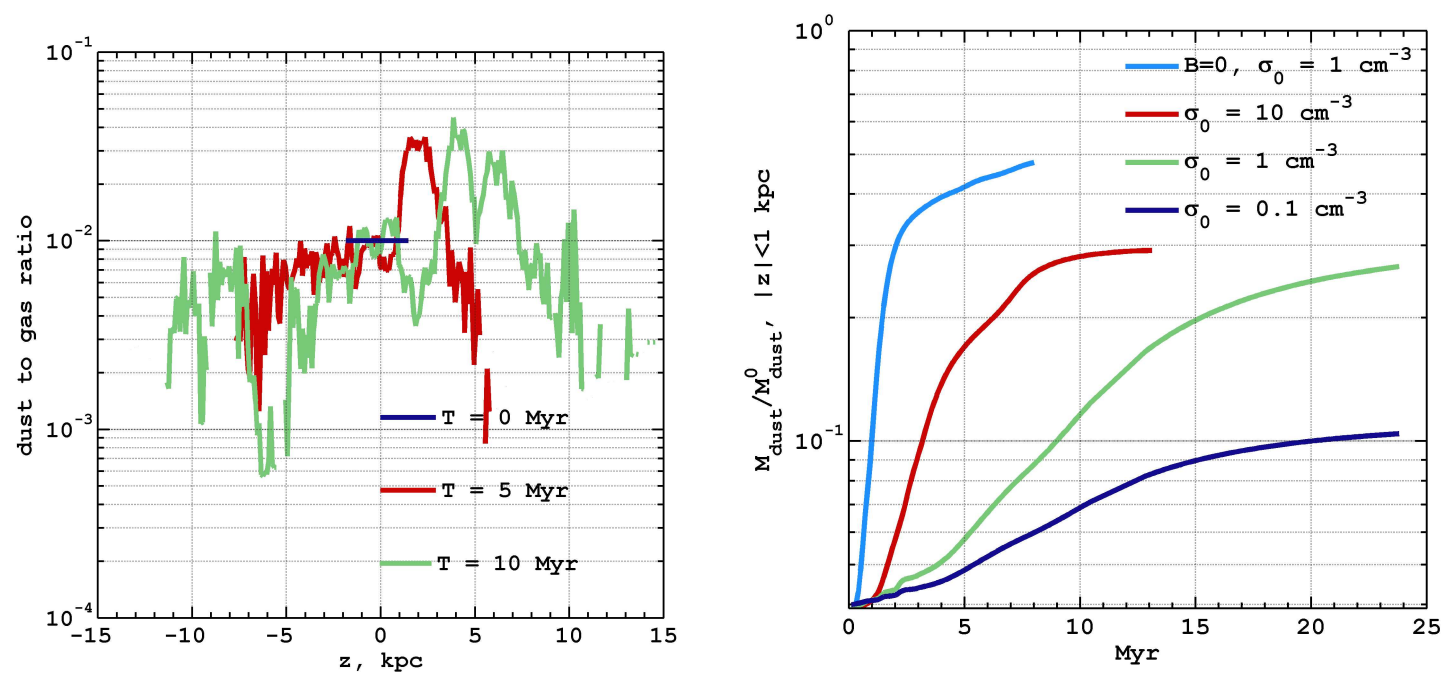

Figure 3: Left: gas-to-dust ratio along the vertical direction at $t=0$ (blue line), $t=5$ (red line) and 10 (green) Myr. Right: fraction of dust mass above $1 \mathrm{kpc}$ : light blue $-\beta=0$ and $\sigma_{0}=1 \mathrm{~cm}^{-3}$; in other simulations $\beta=1$ : red $-\sigma_{0}=10 \mathrm{~cm}^{-3}$, green $-\sigma_{0}=1 \mathrm{~cm}^{-3}$ and dark blue $-\sigma_{0}=0.1 \mathrm{~cm}^{-3}$.

The dust mass loss rate varies from model to model, however the two regimes are a common feature.

- Under the action of SNe shocks magnetic lines bend with a considerable growth of the vertical component in a few dynamical times. It works as an additional efficient mechanism accelerating charged dust particles upwards.

\section{Summary}

Multiple supernovae explosions in the galactic thin stellar disc drive gas and dust into the halo. A large amount of dust can be transported upto $10 \mathrm{kpc}$ in $10-20 \mathrm{Myr}$. Ram pressure from supernovae explosions reorganizes magnetic field structure such that it efficiently accelerates dust grains. The dust outflow rate strongly depends on the plasma- $\beta$ parameter and gas density in the disc. The estimated dust mass loss rate is in the range from $10^{-3} \mathrm{M}_{\odot} \mathrm{yr}^{-1}$ up to $3 \times 10^{-2} \mathrm{M}_{\odot} \mathrm{yr}^{-1}$. Such a high rate is kept though on short time scales $2-10 \mathrm{Myr}$.

\section{References}

[1] H.P. Gail et al., Stardust from Asymptotic Giant Branch Stars, ApJ 698, 1136 (2010)

[2] E. Dwek, The Evolution of the Elemental Abundances in the Gas and Dust Phases of the Galaxy, ApJ 501, 643 (1998)

[3] D. W. Silvia et al., Numerical Simulations of Supernova Dust Destruction. II. Metal-enriched Ejecta Knots, ApJ 748, 12 (2012)

[4] J. A. Irwin, Madden, S. C., Discovery of PAHs in the halo of NGC 5907, A\& A 445, 123 (2006) 
[5] P. Kamphuis et al., A dust component 2 kpc above the plane in NGC 891, A\& A 47, 1 (2007)

[6] J. C. Howk, B. D. Savage, Extraplanar Dust in the Edge-On Spiral NGC 891, AJ 114, 2463 (1997)

[7] B. W. Holwerda, et al., An Extended Dust Disk in a Spiral Galaxy: An Occulting Galaxy Pair in the ACS Nearby Galaxy Survey Treasury, AJ 137, 3000 (2009)

[8] B. Ménard et al., Measuring the galaxy-mass and galaxy-dust correlations through magnification and reddening, MNRAS, 405, 1025 (2010)

[9] de Avillez, M. A. and Breitschwerdt, D., Global dynamical evolution of the ISM in star forming galaxies. I. High resolution 3D simulations: Effect of the magnetic field, A \& A, 436, 585 (2005) 